\title{
Impact of pericardial adhesions on diastolic function as assessed by vortex formation time, a parameter of transmitral flow efficiency
}

\author{
Panupong Jiamsripong ${ }^{1}$, Mohsen S Alharthi ${ }^{1}$, Anna M Calleja ${ }^{1}$, Eileen M McMahon ${ }^{1}$, Minako Katayama ${ }^{1}$,
} John Westerdale ${ }^{2}$, Michele Milano², Jeffrey J Heys ${ }^{3}$, Farouk Mookadam', Marek Belohlavek ${ }^{1 *}$

\begin{abstract}
Background: Pericardial adhesions are a pathophysiological marker of constrictive pericarditis (CP), which impairs cardiac filling by limiting the total cardiac volume compliance and diastolic filling function. We studied diastolic transmitral flow efficiency as a new parameter of filling function in a pericardial adhesion animal model. We hypothesized that vortex formation time (VFT), an index of optimal efficient diastolic transmitral flow, is altered by patchy pericardial-epicardial adhesions.

Methods: In 8 open-chest pigs, the heart was exposed while preserving the pericardium. We experimentally simulated early pericardial constriction and patchy adhesions by instilling instant glue into the pericardial space and using pericardial-epicardial stitches. We studied left ventricular (LV) function and characterized intraventricular blood flow with conventional and Doppler echocardiography at baseline and following the experimental intervention.

Results: Significant decreases in end-diastolic volume, ejection fraction, stroke volume, and late diastolic filling velocity reflected the effects of the pericardial adhesions. The mean VFT value decreased from $3.61 \pm 0.47$ to $2.26 \pm 0.45$ $(\mathrm{P}=0.0002)$. Hemodynamic variables indicated the inhibiting effect of pericardial adhesion on both contraction (decrease in systolic blood pressure and $+\mathrm{dP} / \mathrm{dt}$ decreased) and relaxation (decrease in the magnitude of $-\mathrm{dP} / \mathrm{dt}$ and prolongation of Tau) function.

Conclusion: Patchy pericardial adhesions not only negatively impact LV mechanical functioning but the decrease of VFT from normal to suboptimal value suggests impairment of transmitral flow efficiency.
\end{abstract}

\section{Background}

Left ventricular (LV) diastolic filling and its alterations are important indicators of overall cardiac health status [1-4] and contribute to early diagnosis of cardiovascular disease $[1,5]$. We have shown by echocardiographic particle imaging velocimetry [6], and other investigators have demonstrated by magnetic resonance imaging [7], that transmitral flow can produce an intraventricular rotational body of fluid referred to as a vortex ring [8-10]. This vortex supports more efficient fluid transport as compared to a straight jet alone [11,12]. Gharib

\footnotetext{
* Correspondence: belohlavek.marek@mayo.edu

'Translational Ultrasound Research Laboratory, Division of Cardiovascular

Diseases, Mayo Clinic, Scottsdale, Arizona, USA

Full list of author information is available at the end of the article
}

et al. [13] derived a dimensionless index that quantitatively characterizes optimal fluid dynamic conditions for vortex formation from the duration of flow through an orifice with known diameter and, thus, referred to the index as vortex formation time (VFT). VFT measurement, when applied to diastolic filling, suggests an efficient diastolic transmitral flow if the resulting value is within an optimal range from 3.5 to 4.5 [13]. We have demonstrated that when LV filling is altered, such as during increased LV afterload, the resulting mean VFT value in fact shifts out of the optimal range [14].

Development of pericardial adhesions is a pathophysiological marker of constrictive pericarditis (CP), which impairs cardiac filling by limiting the total cardiac volume compliance $[15,16]$. The current study focuses on
C Biomed Central 
the diastolic filling and VFT parameters and its alteration in an animal model of pericardial adhesions [17]. Capitalizing on existing data from our previous studies we hypothesized that experimentally induced pericardial adhesions will be hemodynamically accompanied not only by impairment in conventional Doppler flow characteristics but also by decreased transmitral flow efficiency, as assessed by VFT.

\section{Materials and methods} Animal Preparation

The study was approved by the Mayo Clinic Institutional Animal Care and Use Committee. Eleven pigs weighing 40 to $45 \mathrm{~kg}$ were anesthetized with inhalation of $1.5 \%$ isoflurane and mechanically ventilated with a mechanical ventilator (Ohmeda 7800 Ventilator; Datex-Ohmeda Inc, Madison, Wisconsin), and blood gases were periodically checked. Pressures in the LV and ascending aorta were measured with high-fidelity catheters (Millar Instruments, Houston, TX) placed via internal carotid artery cannulation. Internal jugular vein cannulation allowed administration of fluids and medications. After median sternotomy, the heart was exposed, sparing the pericardium.

\section{Experimental Intervention}

We have described the pericardial adhesion model in detail previously [17] (Figure 1). Briefly, pericardial-epicardial adhesions were induced with an ethyl-cyanoacrylate glue (Super Glue ${ }^{\mathrm{Tx}}$; Pacer Technology, LLC, Rancho Cucamonga, California). Cyanoacrylate instillation induced patchy adhesions and a thickened shell that encompassed approximately $70 \%$ of the LV pericardial surface.

\section{Hemodynamic Data Analysis}

LV end-diastolic pressure (LVEDP) was timed to the $\mathrm{R}$-wave peak on the synchronous electrocardiographic (ECG) tracing. Besides peak positive $\mathrm{dP} / \mathrm{dt}(+\mathrm{dP} / \mathrm{dt})$ and peak negative $\mathrm{dP} / \mathrm{dt}(-\mathrm{dP} / \mathrm{dt})$, the time constant of $\mathrm{LV}$ pressure decay during the isovolumic relaxation period (Tau) was ascertained by using a validated zero-asymptote model [18].

\section{Echocardiographic Image Acquisition}

An Acuson Sequoia C512 ultrasound system (Siemens Medical Solutions Inc, Mountain View, California) equipped with an $8 \mathrm{~V} 3 \mathrm{C}$ transducer set to $3.5 \mathrm{MHz}$ was used to acquire echocardiographic images. The transducer was placed on the pericardium and acoustically coupled with a small amount of gel. Ultrasound scans for each pig included: 1) apical 2-chamber and 4-chamber views for measurements of LV cardiac output, end-diastolic volume (EDV), end-systolic volume, stroke volume, and ejection fraction; 2) Doppler spectral flow for peak early (E-wave) and atrial (A-wave) filling velocities; and 3) tissue Doppler for peak velocities of the mitral annulus in early (E') phases of LV filling (measured at the basal medial portion of the mitral annulus), and the dimensionless ratio E/E' was computed $[19,20]$. For each scan, 3 cardiac cycles were acquired at a frame rate of $60-70 \mathrm{~Hz}$.

\section{VFT Definition}

Transmitral flow can produce an intraventricular rotating fluid mass referred to as a vortex ring which supports a more efficient fluid transport compared to a straight jet alone $[11,12]$. The vortex ring minimizes
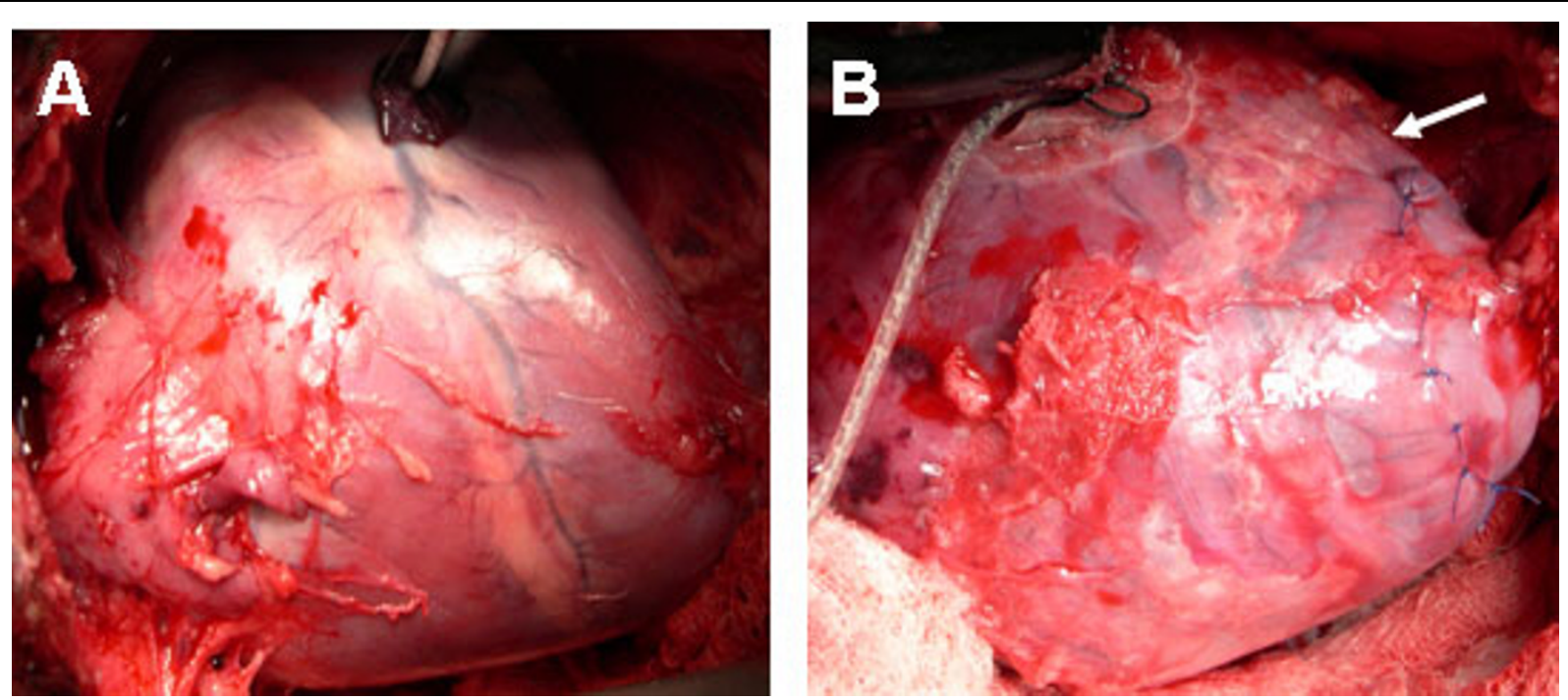

Figure 1 A) Heart with normal pericardium at baseline; B) Heart with stitched and glued pericardium. The arrow points at visible adhesions. 
energy dissipation during fluid ejection through an orifice [21] and optimizes to facilitate the efficiency of fluid transport. The formation of abnormal vortices also relates to the underlying fluid dynamics in LV dysfunction and abnormal LV loading condition [13,22]. Therefore, vortex flow may offer a novel index for blood transport efficiency and may be of incremental value as a marker of cardiac disease. Gharib and colleagues [13] derived the VFT formula, which we refer to as "conventional". The formula is based on LV function, mitral orifice diameter, and diastolic filling flow velocities, ie, parameters that can be measured noninvasively by echocardiography:

$$
\mathrm{VFT}=\frac{4(1-\beta) \alpha^{3}}{\pi} \times \mathrm{EF},
$$

where $\beta$ is the fraction of the stroke volume provided by the atrial component of LV filling and was calculated as the $\mathrm{A}$-wave area divided by the sum of the A-wave and E-wave areas [13]. In our study, we estimated $\beta$ by using the peak magnitude of the A-wave divided by the sum of the A-wave and E-wave peak magnitudes. The symbol $\alpha$ represents an LV geometry parameter defined as $\alpha=\frac{\mathrm{EDV}^{1 / 3}}{\mathrm{D}}$. The $\alpha$ parameter incorporates measurements of EDV in $\mathrm{mL}$ and mitral orifice diameter (D) in $\mathrm{cm}$. The latter has been obtained in our study by averaging the largest mitral orifice diameters obtained during early diastolic filling in the 4-chamber and 2- or 3-chamber apical views.

However, vortex ring formation during diastole can also be defined as [22]:

$$
\mathrm{VFT}=\frac{\overline{\mathrm{U}_{\mathrm{t}}}}{\mathrm{D}} \times \mathrm{T},
$$

where $\overline{U_{t}}(\mathrm{~cm} / \mathrm{s})$ is time-averaged velocity of the fluid flow, $\mathrm{T}$ is the duration of fluid ejection, and $\mathrm{D}(\mathrm{cm})$ is the diameter of the mitral annulus obtained in our study as the mean of the largest diameters measured during early diastolic filling in the 2, 3, or 4-chamber apical views [13]. Because the vortex ring of interest forms in the early phase of diastolic filling, the term $\overline{\mathrm{U}_{\mathrm{t}}} \times \mathrm{t}$ can be measured echocardiographically using the time-velocity integral of the E-wave, ie, $\mathrm{TVI}_{\mathrm{E}}(\mathrm{cm})$. This leads to a simple definition of vortex formation time (ie, VFTs), which has been used in our study as follows:

$$
\mathrm{VFTs}=\frac{\mathrm{TVI}_{\mathrm{E}}}{\mathrm{D}} .
$$

We used this formula because of its practicality and we believe that this formula is a more convenient and more practical implication.

\section{Data Analyses}

Data were expressed as means \pm standard deviation. Hemodynamic, $2 \mathrm{D}$, and Doppler echocardiographic data, as well as strain and strain rate (SR) measurements were obtained at baseline and during intervention. These data were compared using a 2-tailed paired $t$ test. The relationship between different continuous variables was analyzed using single and multiple regression analyses. All differences were considered significant for $\mathrm{P}<$ 0.05 .

\section{Results}

Data were obtained from 8 animals and were used to induce the pericardial adhesion model [14] and generate the results of this study.

\section{Hemodynamic Parameters}

Hemodynamic results are summarized in Table 1. Heart rate did not change. Systolic blood pressure decreased after intervention, while diastolic blood pressure and LV end-diastolic pressure did not significantly change. However, magnitudes of $+\mathrm{dP} / \mathrm{dt}$ and $-\mathrm{dP} / \mathrm{dt}$ decreased significantly while Tau has been prolonged, suggesting an inhibiting effect of the experimental pericardial adhesions on the rate of LV contraction and relaxation. These changes coincided with a decrease in cardiac output. We did not observe respiratory variations or the characteristic dip and plateau of the LV pressure tracing.

\section{Echocardiographic Parameters}

Conventional echocardiographic measurements of LV filling and mechanical function are summarized in Table 2. Peak A-wave (but not E-wave) velocity decreased and

Table 1 Hemodynamic Parameters

\begin{tabular}{lccc}
\hline Variable & Baseline & Adhesions & $\boldsymbol{P}$ value \\
\hline $\mathrm{BPs}(\mathrm{mm} \mathrm{Hg})$ & $111.59 \pm 10.55$ & $94.66 \pm 20.51$ & 0.0382 \\
$\mathrm{BPd}(\mathrm{mm} \mathrm{Hg})$ & $78.10 \pm 7.70$ & $66.54 \pm 21.91$ & 0.1276 \\
$\mathrm{BPm}(\mathrm{mm} \mathrm{Hg})$ & $89.26 \pm 8.37$ & $75.91 \pm 21.33$ & 0.0842 \\
$\mathrm{HR}(\mathrm{beats} / \mathrm{min})$ & $77.50 \pm 7.91$ & $84.88 \pm 9.43$ & 0.2230 \\
$\mathrm{CO}(\mathrm{mL} / \mathrm{min})$ & $2.66 \pm 0.90$ & $1.44 \pm 0.48$ & 0.0005 \\
$\mathrm{LVEDP}(\mathrm{mm} \mathrm{Hg})$ & $7.50 \pm 3.78$ & $9.29 \pm 4.76$ & 0.4239 \\
$+\mathrm{dP} / \mathrm{dt}(\mathrm{mm} \mathrm{Hg} / \mathrm{sec})$ & $1096.50 \pm 236.41$ & $795.50 \pm 155.03$ & 0.0005 \\
$-\mathrm{dP} / \mathrm{dt}(\mathrm{mm} \mathrm{Hg} / \mathrm{sec})$ & $-1490.63 \pm 198.60$ & $-1044.75 \pm 219.31$ & 0.0002 \\
Tau $(\mathrm{msec})$ & $42.46 \pm 9.06$ & $70.66 \pm 34.81$ & 0.0470
\end{tabular}

BPd, blood pressure diastolic; BPm, mean arterial blood pressure; BPs, blood pressure systolic; $\mathrm{CO}$, cardiac output; $+\mathrm{dP} / \mathrm{dt}$, maximum positive LV pressure change; $-d P / d t$, maximum negative LV pressure change; HR, heart rate; LVEDP, left ventricular end-diastolic pressure; Tau, the time constant of isovolumic relaxation. 
Table 2 Echocardiographic Parameters

\begin{tabular}{lccc}
\hline Variable & Baseline & Intervention & $\boldsymbol{P}$ value \\
\hline E $(\mathrm{m} / \mathrm{s})$ & $0.49 \pm 0.16$ & $0.44 \pm 0.04$ & 0.3228 \\
$A(\mathrm{~m} / \mathrm{s})$ & $0.55 \pm 0.12$ & $0.42 \pm 0.13$ & 0.0342 \\
E/A & $0.92 \pm 0.28$ & $1.12 \pm 0.32$ & 0.1162 \\
E/E' & $5.21 \pm 1.60$ & $4.03 \pm 1.15$ & 0.1310 \\
EDV $(\mathrm{mL})$ & $60.73 \pm 18.11$ & $42.30 \pm 11.13$ & 0.0008 \\
ESV $(\mathrm{mL})$ & $25.50 \pm 7.65$ & $24.31 \pm 6.83$ & 0.5223 \\
$S V(\mathrm{~mL})$ & $34.49 \pm 10.55$ & $17.22 \pm 4.95$ & 0.0002 \\
EF $(\%)$ & $57.03 \pm 2.44$ & $40.66 \pm 6.20$ & 0.0001 \\
\hline
\end{tabular}

A, peak transmitral flow velocities at late filling phases; $E$, peak transmitral flow velocities at early filling phase; $E^{\prime}$, early diastolic myocardial peak velocity septal annulus; EDV, left ventricular end-diastolic volume; EF, ejection fraction; ESV, left ventricular end-systolic volume; SV, stroke volume.

the $\mathrm{E} / \mathrm{A}$ ratio showed an increasing trend, suggesting impairment in LV preload. E/E' did not change. While end-systolic volume did not change either, EDV decreased significantly, resulting in a post-intervention drop in cardiac output, stroke volume, and ejection fraction.

\section{Vortex Formation Parameters}

The mean values of $\mathrm{D}$, when compared between baseline and intervention, remained nearly identical (Table 3). $\mathrm{TVI}_{\mathrm{E}}$ decreased and resulted in a significant decrease in VFT after the intervention (Figure 2).

\section{Discussion}

To our knowledge, this study shows for the first time that experimentally simulated early (patchy) formation of a noncompliant pericardium with adhesions negatively affects transmitral flow efficiency, as expressed by a shift in VFT values out of their optimal range.

\section{Model of Pericardial Thickening and Adhesions}

Our animal model produced areas of pliable and rigid pericardium (based on direct palpation during the openchest study) and strong pericardial-epicardial adhesions. In a fully developed model of $\mathrm{CP}$, the encasement of the heart by a rigid shell of noncompliant pericardium results in characteristic pathophysiologic effects, including impaired diastolic filling of the ventricles [23].

Hemodynamic parameters (Table 1) and functional parameters (Table 2) were significantly affected by the experimentally induced pericardial adhesions. Septal

\section{Table 3 Vortex Formation Parameters}

\begin{tabular}{cccc}
\hline Variable & Baseline & Intervention & $P$ value \\
\hline$D(\mathrm{~cm})$ & $1.76 \pm 0.18$ & $1.78 \pm 0.15$ & 0.7660 \\
$T V I_{E}$ & $6.31 \pm 0.99$ & $4.05 \pm 0.85$ & 0.0004 \\
$V F T$ & $3.61 \pm 0.47$ & $2.26 \pm 0.45$ & 0.0002 \\
\hline
\end{tabular}

$\mathrm{D}$, diameter of mitral valve orifice; $\mathrm{TVI}_{\mathrm{E}}$, time-velocity integral of the $\mathrm{E}$-wave; VFT, vortex formation time.

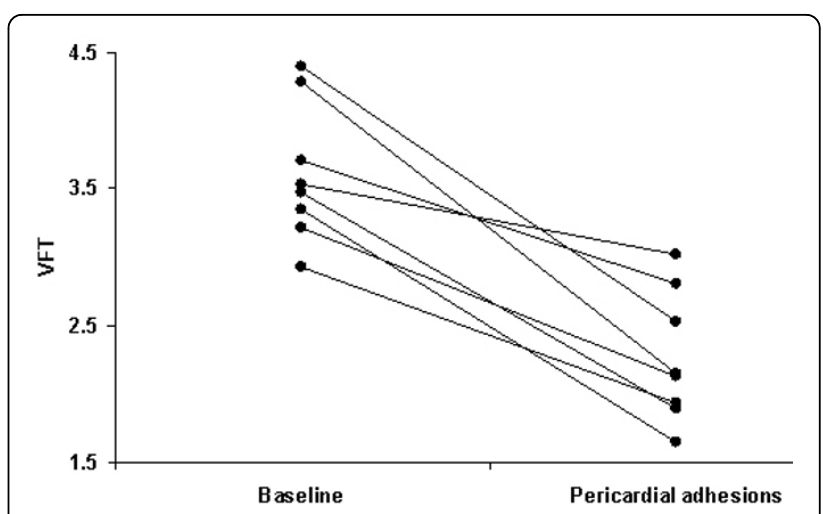

Figure 2 Change of vortex formation time (VFT) value from baseline to intervention.

bounce was seen in 5 out of 8 animals. However, respiratory variation of the E- wave was not consistently present nor was a high E/A ratio. The lack of these diagnostic characteristics was likely due to controlled respiration in the sedated and mechanically-ventilated animals.

\section{Systolic and Diastolic Function Following Induction of Pericardial Adhesions}

In our model, LV function was impaired in its mechanical performance by patchy pericardial-epicardial adhesions and spotty stiffening or hardening of the pericardium led to impairment of both systolic and diastolic myocardial function. The observed decreases in ejection fraction, stroke volume, cardiac output, and $+\mathrm{dP} / \mathrm{dt}$ are consistent with impairment in systolic function, but the mechanism of reduced ejection performance may be secondary to impaired ventricular filling, especially in mid- and late diastole (reduced preload) [15].

The decrease in the absolute value of $-\mathrm{dP} / \mathrm{dt}$ and an increase in Tau are consistent with impairment in diastolic function. Impairments in LV filling (due to pericardial constriction) [24,25] are the most likely mechanisms of diastolic dysfunction. The impairment of LV filling typically presents at early diastole by a high $\mathrm{E}$ velocity and E/A ratio. But due to the acute study, some conventional echocardiographic findings, such as a high E/A ratio and significant transmitral or tricuspid E-wave respiratory variations, were absent.

Although the possibility of injury and its effect on LV function cannot be excluded, Kaplan et al [26] showed that ethyl-cyanoacrylate tissue glue does not cause foreign body, inflammatory, necrotic, or other histopathologic reactions when used for bonding of cardiovascular tissues. Also, the pericardial-epicardial stitches were placed very carefully, with no subsequent bleeding or apparent injury. 


\section{Changes in VFT and its Component Parameters with Pericardial Adhesions}

LV diastolic filling plays an important role in cardiac function and its optimal means of cardiac efficiency [13]. LV diastolic function can be assessed by invasive and non-invasive methods. Doppler flow echocardiography is the most practical routine clinical approach of a non-invasive method for evaluating LV diastolic function. Doppler is performed to obtain mitral inflow velocities during diastole which has peak E (early diastolic) and A (late diastolic) [27]. E wave is predominant LV filling from the LA to the LV. The flow of blood during $\mathrm{E}$ wave occurs from the rapid pressure drop in LV by accelerating through the mitral valve and has been observed to induce formation of a rotating fluid of blood called a vortex ring [13].

The diastolic vortex ring acts as a kinetic energy reservoir that facilitates propulsion of blood in systole [21], contributes to blood redirection into the outflow tract and aorta [28,29], and helps in preventing blood stagnation within the LV apex [29]. Based on mathematical modeling, the vortex ring may exert a force on the mitral valve, thus contributing to its timely closure [30]. Premature disintegration of the vortex and dissipation of the stored energy eliminates the beneficial effects of vortex formation and may necessitate an increase in the physical work generated by the cardiac muscle, thus augmenting the oxygen consumption, which in turn reduces the efficiency of the heart [31]. The process of vortex ring formation during diastole can serve as an indicator of cardiac health [13].

The rapid development of the adhesions in our study impairs both systolic and diastolic myocardial function. Consequently, as our study shows, this alteration also negatively impacts conditions for vortex ring formation, (indicated by the mean VFT value shift to below the optimal range) and implies the loss of optimal hemodynamic efficiency during LV filling [32].

\section{Clinical Implications}

This is a starting point towards further investigations of VFT in chronic pericarditis both in animal experiments and clinical practice. Diagnosis of early stages of CP can be challenging because of variable hemodynamic presentations and the difficulty of distinguishing CP from other diseases with similar symptoms and signs [33]. VFT may supersede conventional parameters of cardiac mechanical or hemodynamic function, because it is a disease stage independent factor as well as a patient effects independent parameter [13]. VFT is a dimensionless index that is easy to perform with simple echocardiographic principles, it is a dimensionless index defined on a universal timescale [22] and hence has practical advantages. As we have shown, our animal model replicates pericardial adhesion and the VFT parameter contributes to a conceptually novel interpretation of cardiac function (ie, efficiency of diastolic blood transport) in such a situation.

\section{Limitations}

Spreading the tissue glue within the pericardial space was a manual process, which introduced variations in the distribution of adhesions and pericardial stiffening. However, despite the variability in this process, we observed a consistent and statistically significant decrease in VFT. We cannot conclude that this animal model of pericardial adhesion is the same as physiology in chronic pericarditis. We plan on conducting further studies, both acute and chronic, to continue the development of the animal model and to characterize its functional and histopathologic effects on myocardium. There is a slight inconsistency between the optimal VFT value range considered for experimental in vitro setting and clinical settings; the latter has a somewhat broader range [13]. Thus, VFT needs to be broadly investigated in normal and disease conditions both in the clinical and experimental animal setting.

\section{Conclusion}

In our animal model, patchy pericardial stiffening and pericardial-epicardial adhesions resulted in systolic and diastolic dysfunction. However besides the mechanical effect, we found also detrimental effect of pericardial adhesion on diastolic vortex function, a decrease in the VFT value, suggesting impairment in the efficiency of diastolic filling.

\section{Acknowledgements \\ We thank Theresa Lombari for veterinary assistance and Danielle R. Wright for secretarial help. We thank Siemens Medical Solutions, Inc, for loaning us the ultrasound system, and Helene Houle, RDMS, for technical assistance. This study was sponsored in parts by a research grant from Siemens Medical Solutions, Inc., and by a collaborative Arizona State University - Mayo Clinic Seed Grant.}

\section{Author details}

${ }^{1}$ Translational Ultrasound Research Laboratory, Division of Cardiovascular Diseases, Mayo Clinic, Scottsdale, Arizona, USA. ${ }^{2}$ Department of Mechanical Engineering, Arizona State University, Tempe, AZ, USA. ${ }^{3}$ Chemical and Biological Engineering, Montana State University, Bozeman, MT, USA.

\section{Authors' contributions}

JP conceived the study and participated in the design and creation, performed detailed statistical analyses, and drafted manuscript (original and revision). MA critically reviewed study and manuscript. AC critically reviewed study and manuscript. EM critically reviewed study and manuscript. MK critically reviewed the manuscript. JW critically reviewed VFT analyses. MM critically reviewed VFT analyses. JH critically reviewed VFT analyses. FM critical review of manuscript. MB participated in design and creation of study and critically reviewed statistical analyses and manuscript. All authors read and approved the final manuscript.

\section{Competing interests}

The authors declare that they have no competing interests. 
Received: 2 July 2010 Accepted: 22 September 2010

Published: 22 September 2010

\section{References}

1. Ohno $M$, Cheng $C P$, Little WC: Mechanism of altered patterns of left ventricular filling during the development of congestive heart failure. Circulation 1994, 89(5):2241-2250.

2. Vasan RS, Larson MG, Benjamin EJ, Evans JC, Reiss CK, Levy D: Congestive heart failure in subjects with normal versus reduced left ventricular ejection fraction: prevalence and mortality in a population-based cohort. J Am Coll Cardiol 1999, 33(7):1948-1955.

3. Yellin EL, Meisner JS: Physiology of diastolic function and transmitral pressure-flow relations. Cardiol Clin 2000, 18(3):411-433.

4. Zile MR, Brutsaert DL: New concepts in diastolic dysfunction and diastolic heart failure: Part I: diagnosis, prognosis, and measurements of diastolic function. Circulation 2002, 105(11):1387-1393.

5. Hasegawa H, Little WC, Ohno M, Brucks S, Morimoto A, Cheng HJ, Cheng CP: Diastolic mitral annular velocity during the development of heart failure. J Am Coll Cardiol 2003, 41(9):1590-1597.

6. Sengupta PP, Korinek J, Belohlavek M, Narula J, Vannan MA, Jahangir A, Khandheria BK: Left ventricular structure and function: basic science for cardiac imaging. J Am Coll Cardiol 2006, 48(10):1988-2001.

7. Kilner PJ, Yang GZ, Wilkes AJ, Mohiaddin RH, Firmin DN, Yacoub MH: Asymmetric redirection of flow through the heart. Nature 2000, 404(6779):759-761.

8. Kheradvar A, Gharib M: Influence of ventricular pressure drop on mitral annulus dynamics through the process of vortex ring formation. Ann Biomed Eng 2007, 35(12):2050-2064.

9. Collier E, Hertzberg J, Shandas R: Regression analysis for vortex ring characteristics during left ventricular filling. Biomed Sci Instrum 2002, 38:307-311.

10. Shandas R, Gharib M, Liepman D, Shiota T, Sahn DJ: Experimental studies to define the geometry of the flow convergence region. Laser Doppler particle tracking and color Doppler imaging. Echocardiography 1992, 9(1):43-50.

11. Dabiri JO, Gharib M: The role of optimal vortex formation in biological fluid transport. Proc Biol Sci 2005, 272(1572):1557-1560.

12. Krueger PS, Gharib M: The significance of vortex ring formation to the impulse and thrust of a starting jet. Physics of Fluids 2003, 15:1271-1281.

13. Gharib M, Rambod E, Kheradvar A, Sahn DJ, Dabiri JO: Optimal vortex formation as an index of cardiac health. Proc Natl Acad Sci USA 2006, 103(16):6305-6308.

14. Jiamsripong P, Alharthi MS, Calleja AM, MCMahon EM, Mookadam F, Khandheria BK, Belohlavek M: Quantification of Left Ventricular Twisting Mechanics by Velocity Vector Imaging in an Animal Model of Pericardial Adhesions. Ultrasound in Medicine and Biology 2009.

15. Nishimura RA: Constrictive pericarditis in the modern era: a diagnostic dilemma. Heart 2001, 86(6):619-623.

16. Maisch $B$, Seferovic PM, Ristic AD, Erbel R, Rienmuller $R$, Adler $Y$, Tomkowski WZ, Thiene G, Yacoub MH: Guidelines on the diagnosis and management of pericardial diseases executive summary; The Task force on the diagnosis and management of pericardial diseases of the European society of cardiology. Eur Heart J 2004, 25(7):587-610.

17. Alharthi MS, Jiamsripong P, Calleja A, Sengupta PP, McMahon EM, Khandheria B, Tajik AJ, Belohlavek M: Selective echocardiographic analysis of epicardial and endocardial left ventricular rotational mechanics in an animal model of pericardial adhesions. Eur J Echocardiogr 2009, 10(3):357-362.

18. Nishimura RA, Schwartz RS, Tajik AJ, Holmes DR Jr: Noninvasive measurement of rate of left ventricular relaxation by Doppler echocardiography. Validation with simultaneous cardiac catheterization. Circulation 1993, 88(1):146-155.

19. Quinones MA: Assessment of diastolic function. Prog Cardiovasc Dis 2005, 47(5):340-355

20. Oh JK, Hatle L, Tajik AJ, Little WC: Diastolic heart failure can be diagnosed by comprehensive two-dimensional and Doppler echocardiography. J Am Coll Cardiol 2006, 47(3):500-506.

21. Pedrizzetti $G$, Domenichini F: Nature optimizes the swirling flow in the human left ventricle. Phys Rev Lett 2005, 95(10):108101/108101-108101/ 108104.
22. Gharib M, Rambod E, Shariff K: A universal timescale for vortes ring formation. J Fluid Mech 1998, 360:121-140.

23. Myers RB, Spodick DH: Constrictive pericarditis: clinical and pathophysiologic characteristics. Am Heart J 1999, 138(2 Pt 1):219-232.

24. Rademakers FE, Buchalter MB, Rogers WJ, Zerhouni EA, Weisfeldt ML, Weiss JL, Shapiro EP: Dissociation between left ventricular untwisting and filling. Accentuation by catecholamines. Circulation 1992, 85(4):1572-1581.

25. Dong SJ, Hees PS, Siu CO, Weiss JL, Shapiro EP: MRI assessment of LV relaxation by untwisting rate: a new isovolumic phase measure of tau. Am J Physiol Heart Circ Physiol 2001, 281(5):H2002-2009.

26. Kaplan M, Bozkurt S, Kut MS, Kullu S, Demirtas MM: Histopathological effects of ethyl 2-cyanoacrylate tissue adhesive following surgical application: an experimental study. Eur J Cardiothorac Surg 2004, 25(2):167-172.

27. Nagueh SF, Appleton CP, Gillebert TC, Marino PN, Oh JK, Smiseth OA, Waggoner AD, Flachskampf FA, Pellikka PA, Evangelista A: Recommendations for the evaluation of left ventricular diastolic function by echocardiography. J Am Soc Echocardiogr 2009, 22(2):107-133.

28. Sengupta PP, Khandheria BK, Korinek J, Jahangir A, Yoshifuku S, Milosevic I, Belohlavek M: Left ventricular isovolumic flow sequence during sinus and paced rhythms: new insights from use of high-resolution Doppler and ultrasonic digital particle imaging velocimetry. J Am Coll Cardiol 2007. 49(8):899-908

29. Domenichini F, Pedrizzetti $G$, Baccani B: Three-dimensional filling flow into a model left ventricle. J Fluid Mech 2005, 539:179-198.

30. Reul H, Talukder N, Muller EW: Fluid mechanics of the natural mitral valve. J Biomech 1981, 14(5):361-372.

31. Pedrizzetti $G$, Domenichini F: Nature optimizes the swirling flow in the human left ventricle. Phys Rev Lett 2005, 95(10):108101.

32. Nishimura RA, Tajik AJ: Evaluation of diastolic filling of left ventricle in health and disease: Doppler echocardiography is the clinician's Rosetta Stone. J Am Coll Cardiol 1997, 30(1):8-18.

33. Ha JW, Oh JK, Ommen SR, Ling LH, Tajik AJ: Diagnostic value of mitral annular velocity for constrictive pericarditis in the absence of respiratory variation in mitral inflow velocity. J Am Soc Echocardiogr 2002, 15(12):1468-1471.

doi:10.1186/1476-7120-8-42

Cite this article as: Jiamsripong et al:: Impact of pericardial adhesions on diastolic function as assessed by vortex formation time, a parameter of transmitral flow efficiency. Cardiovascular Ultrasound 2010 8:42.

\section{Submit your next manuscript to BioMed Central and take full advantage of:}

- Convenient online submission

- Thorough peer review

- No space constraints or color figure charges

- Immediate publication on acceptance

- Inclusion in PubMed, CAS, Scopus and Google Scholar

- Research which is freely available for redistribution 